Revue d'histoire de l'Amérique française

REVUE D.HISTOIRE DE L'AMÉRIQUE FRANÇAISE

RUTHERFORD, Paul, The Making of the Canadian Media.

McGraw-Hill Ryerson Limited, Montréal, New York, 1978. 141 p. \$12.95 relié ; \$5.95 broché.

\title{
Gérard Laurence
}

Volume 34, numéro 2, septembre 1980

URI : https://id.erudit.org/iderudit/303868ar

DOI : https://doi.org/10.7202/303868ar

Aller au sommaire du numéro

Éditeur(s)

Institut d'histoire de l'Amérique française

ISSN

0035-2357 (imprimé)

1492-1383 (numérique)

Découvrir la revue

Citer ce compte rendu

Laurence, G. (1980). Compte rendu de [RUTHERFORD, Paul, The Making of the

Canadian Media. McGraw-Hill Ryerson Limited, Montréal, New York, 1978

141 p. $\$ 12.95$ relié ; $\$ 5.95$ broché.] Revue d'histoire de l'Amérique française,

34(2), 289-293. https://doi.org/10.7202/303868ar d'utilisation que vous pouvez consulter en ligne.

https://apropos.erudit.org/fr/usagers/politique-dutilisation/ 
RUTHERFORD, Paul, The Making of the Canadian Media. McGrawHill Ryerson Limited, Montréal, New York, 1978. 141 p. \$12.95 relié; $\$ 5.95$ broché.

Dans le domaine de l'histoire des médias, les ouvrages canadiens sont trop rares pour que nous puissions passer celui-ci sous silence. Depuis quelques années, sans doute publie-t-on en cette matière de plus en plus de livres ou d'articles, généralement très fouillés et de nature nettement universitaire. Mais ce sont, dans la quasi-totalité des cas, des études sectorielles, des gros plans sur des phénomènes, des aspects ou des périodes plutôt partiels et limités. Le travail de Paul Rutherford, professeur au département d'histoire de l'université de Toronto, se démarque assez nettement des publications de ce genre. C'est, en effet, un long travelling latéral qu'effectue l'auteur, nous faisant remonter, en moins de 150 pages, les 225 années de l'histoire des médias canadiens des origines à ces dernières années. Mais encore faut-il sensiblement nuancer cette appréciation globale.

Le long travelling latéral ne constitue pas, à la vérité, un plan unique mais trois plans successifs correspondant aux trois chapitres de l'ouvrage. Le premier chapitre, "The Rise of the Newspaper», couvre les années allant de la conquête britannique à la Confédération. Le second chapitre, "The Golden Age of the Press», nous mène de la Confédération à la période de la crise. Le dernier chapitre, "The Triumph of the Multimedia», traite des cinq dernières décennies, depuis les années 30 jusqu'à la fin des années 70 . Ce qui nous fait parler de trois plans successifs, ce sont le ton et la texture de chacun des chapitres. Le premier est nettement pédagogique et didactique: il raconte avec application l'émergence de la presse écrite au Canada depuis ses débuts laborieux et tardifs au milieu du XVIIIe siècle jusqu'aux premiers signes de ses effets corrosifs sur l'ordre "tory» au milieu du XIXe siècle. Le second chapitre est beaucoup plus analytique et systématique. Cet âge d'or de la presse écrite au Canada est caractérisé par une multiplication et une diversification des titres (d'où la nécessité d'en faire l'inventaire, le classement et l'évaluation), par une accélération considérable des transformations de ce monde de l'information (d'où le besoin d'en circonscrire les raisons, les effets et les caractères), par l'importance, enfin, des quotidiens des grandes villes qui, plus que tout autre publication, subissent l'impact de ces mutations. Le troisième chapitre, quant à lui, est grouillant et plus nette- 
ment partisan, à l'image sans doute de ce dernier demi-siècle où viennent s'entrechoquer les différents moyens de diffusion collective. Ce dernier chapitre a donc des airs d'auberge espagnole; on y trouve de tout, le pire et le meilleur, les banalités les plus usées, mais peut-être nécessaires à rappeler (sur les effets de la télévision, sur la publicité, etc.) et les aperçus les plus intéressants (sur le mythe et la recherche du bien-être et leur relation avec les médias), les évaluations les plus contestables (sur les journaux populaires, le syndicalisme des journalistes, les concentrations, etc.) et les remarques les plus justes (sur les origines de la dépendance parasitaire et atavique du Canada à l'égard des productions étrangères, sur la concurrence des médias entre eux, par exemple).

Ce dernier chapitre achève d'ailleurs de donner un ton sans équivoque à ce livre. L'ouvrage de Paul Rutherford ne constitue pas, à proprement parler, une synthèse de l'histoire des médias canadiens construite selon les lois du genre. L'auteur se défend, d'entrée, d'avoir entretenu une telle prétention. Son intention est clairement énoncée dès l'introduction: il s'agit pour lui de proposer une relecture de l'histoire des médias au Canada, relecture nourrie par les apports des théoriciens de la com-

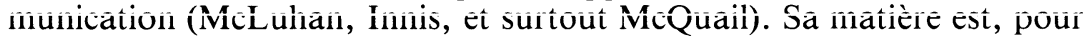
l'essentiel, composée des résultats de travaux antérieurs; mais il ne craint pas, quand nécessaire, de recourir à des sources primaires. Et si l'auteur fait de l'histoire, il ne se cache pas de faire de l'histoire partisane; il se déclare fondamentalement acquis en ce domaine aux conceptions libérales et reconnaît faire une évaluation plutôt conservatrice des médias actuels. Ces choix de départ donnent donc à l'ouvrage plutôt l'aspect d'un essai historique sur les médias canadiens.

Le livre possède les avantages et désavantages du genre. Au chapitre des avantages, il convient de mentionner le survol de la trame événementielle que permet une telle perspective. L'auteur évite ainsi la fastidieuse énumération des noms, dates, titres, faits qui, hachant le discours, constitue trop souvent un handicap pour une appréhension synthétique du phénomène. Non pas que l'évolution des faits soit traitée cavalièrement. L'humus factuel, au contraire, est riche et, à cet égard, ce livre peut, sous certaines réserves, rendre d'excellents services. L'idéal, cependant, est de l'utiliser conjointement avec celui de Kesterton (A History of Journalism in Canada). Second avantage non négligeable, une telle approche permet à l'auteur de risquer des interprétations, des hypothèses, des évaluations, de poser des jugements qui, bien que parfois osés, n'attirent jamais une désapprobation radicale, tout au plus un désaccord nuancé ou un septicisme indulgent. Souvent, même, ces remarques ou ces aperçus sont très toniques et poussent à la réflexion, voire à certaines révisions.

Mais ces avantages ont leurs revers. À vouloir voler trop haut ou trop vite, l'auteur risque souvent des erreurs, des raccourcis ou des oublis. C'est ainsi que Paul Rutherford semble faire la confusion entre la 
«presse du pauvre», née en Angleterre dans la décennie 1810 (le Political Register à $2 \mathrm{~d}$. de Cobbett en fut l'exemple le plus prestigieux) et la «presse populaire» née aux États-Unis dans les années 1830 (avec notamment B. Day et G. Bennett). Sans doute existe-t-il une étroite relation entre ces deux phénomènes puisque les Américains appliquèrent à leurs quotidiens la baisse radicale de prix que les Anglais avaient tentée avec certains de leurs périodiques. Mais cette "presse du pauvre» avait très vite été écrasée, les prix des publications ayant été lourdement grevés par les fameuses «taxes sur le savoir». Ce n'est que lorsque ces taxes furent abolies, à partir de 1855, que put naître en Angleterre une presse véritablement populaire. Et cela nous reporte déjà vingt ans plus tard par rapport aux États-Unis. Il est inexact aussi d'affirmer que le CRTC, désireux de "canadianiser» le câble, obligea les compagnies à supprimer la publicité des émissions en provenance des États-Unis (p. 115). En réalité, le CRTC ne fit que des recommandations en ce sens et n'imposa nulle règle. Ces recommandations qui visaient à encourager l'investissement publicitaire canadien au pays ne furent jamais conçues comme devant constituer une mesure de "canadianisation» du câble. Il y aurait lieu aussi de vérifier, à tout le moins de nuancer, l'affirmation selon laquelle les «penny papers» naquirent au Canada au milieu de la décennie 1830 (p. 29) ou celle qui prétend que la radio devint, dès les années 1930, «a new weapon of considerable potency in the struggle for votes» (p. 105).

Les lacunes et les raccourcis sont peut-être, dans ce type d'ouvrage, plus gênants que les erreurs factuelles de détail. Sans infirmer les démonstrations de l'auteur ils les minent et les rendent parfois plus fragiles. C'est ainsi que la coupure entre le premier chapitre et le second est trop radicale. Pour certains phénomènes, tels la présentation et le contenu d'un quotidien, nous passons sans transition des années $1850 \mathrm{au}$ début des années 1900. C'est ainsi encore que les cinquante ans d'histoire de la radio sont réglés en moins de deux pages (pp. 79-81) et que dans ce laminage sont passées sous silence l'invention et l'exploitation radiophonique du disque, l'arrivée du magnétophone portatif, surtout l'intrusion du transistor. Quasiment oublié aussi - il n'est évoqué qu'une seule fois (p. 89) - le procédé d'impression par of fset qui transforma sensiblement les conditions de publication. Quant aux contenus des programmes de radio ou de télévision il en est fait, en de très rares occasions, de trop rapides allusions. Pourquoi avoir occulté cet aspect essentiel de ces deux médias alors qu'il est consacré aux contenus et à la présentation des journaux de très nombreuses (et souvent fort intéressantes) pages? Il n'est rien dit, en outre, de Radio-Québec pas même nommé, ni du différend Ottawa-Québec en matière de communications, pourtant si exemplaire. Et nous constatons, dressant cette liste nullement exhaustive, que c'est principalement le chapitre trois qui est visé. Nous parlons d'oublis, de raccourcis, de lacunes, alors que ce chapitre se présente comme un fouillis grouillant de trop d'éléments. C'était bien là un risque auquel s'expo- 
sait l'auteur en s'attaquant de cette manière à des problèmes encore si chauds et des phénomènes insuffisamment étudiés à ce jour et trop souvent controversés. Non que l'entreprise, aussi difficile qu'elle soit, eût été impossible à mener. Mais faisant oeuvre d'essayiste Paul Rutherford se lance avec passion dans cette période et ses problèmes alors qu'il aurait fallu procéder avec la plus extrême rigueur et avancer avec la plus grande prudence. C'est dans ce chapitre par exemple que l'on sent le plus nettement les idées plus propres aux Canadiens anglais à l'égard du syndicalisme des journalistes, du problème des concentrations ou encore du contrôle de l'État sur la radio et la télévision. Paradoxalement pourtant on pourrait reprocher à l'auteur de n'avoir esquissé aucune perspective sur les techniques et problèmes d'avenir (satellites, vidéocassettes, vidéodisques, utilisation à d'autres fins de l'écran de télévision, etc.). Nous y sommes engagés et toucher au présent obligeait, inévitablement, à les évoquer. Ce qui n'est pas fait.

Plus fondamentalement enfin pourrait-on contester à l'auteur la manière dont il traite sa thèse centrale. Il cherche essentiellement à montrer comment les médias ont constitué au Canada un ferment de mutation, juuani ainsi le rôle d'une véritabie institution. îi démontre d'abord la manière dont la presse écrite, se retournant contre ceux qui l'avaient introduite, contribua à saper l'ordre «tory». Puis il décrit la façon dont cette presse écrite favorisa l'établissement et la consolidation de la «morale» victorienne qui, jusqu'à la veille de la Seconde Guerre mondiale, concourut largement à l'épanouissement et au développement du Canada. Enfin l'auteur tente d'établir comment, depuis le dernier conflit mondial, les divers moyens de communication de masse alimentent le mythe du bien-être, de la richesse, de la consommation tout en s'en nourrissant. Cette recherche justifierait le droit à la «dissidence», à la dissémination et à la segmentation des messages, à la dépolitisation des médias, etc. La démonstration, pour intéressante qu'elle soit, n'est pas probante car l'auteur semble souvent forcer les faits aux fins mêmes de cette démonstration. Surtout, privilégiant trop systématiquement les médias dans le processus de mutation du Canada au cours de ces deux siècles, il les isole arbitrairement du contexte général et leur accorde de ce fait une importance démesurée dans l'éventail des autres variables et facteurs de transformation. Sans doute ce type de traitement était-il inévitable compte tenu des choix premiers de l'auteur et des limites de l'entreprise. Mais peut-être Paul Rutherford eût-il pu chercher à réduire les distorsions trop évidentes qui en résultent.

En comparaison, les bavures d'édition sont de peu d'importance. On eût aimé pourtant, à la page 47, avoir la date des chiffres de tirage qui nous sont livrés pour les magazines de fin de semaine. Il eût été préférable aussi de corriger les énormes fautes qui figurent dans la plupart des références ou citations en français (p. 17, p. 44, p. 54, p. 59). Enfin, les pages 137 à 141 sont à l'évidence mal titrées. 
Toutes ces réserves, critiques et remarques ne doivent pas, cependant, réduire à rien les mérites de cet ouvrage. L'entreprise était difficile car les études historiques en ce domaine sont encore très lacunaires; pourtant, la période et les domaines couverts sont considérables. Les historiens auraient tort, cependant, de faire la fine bouche. À défaut d'une recherche originale, il y a là un livre très personnel, très vivifiant et animé d'une passion qui ne réduit jamais le sérieux du propos. 